\title{
Utilization of Dialysis Membrane for Biosensor Applications by UV Spectrophotometric Detection
}

\section{Yekbun Adiguzel ${ }^{1, *}$}

1 Department of Biophysics, Faculty of Medicine, Istanbul Kemerburgaz University, Mahmutbey Dilmenler Caddesi, No:26, 34217 Bagcilar, Istanbul, Turkey; E-Mails:

yekbun.adiguzel@kemerburgaz.edu.tr

* Author to whom correspondence should be addressed; E-Mail:

yekbun.adiguzel@kemerburgaz.edu.tr;

Tel.: +90-212-604-0100/1427; Fax: +90-212-445-8171.

Published: 1 June 2014

\begin{abstract}
Investigating original applications of conventional media for biosensors field resides prospective developments in health-care services. As an example, colorimetric test strips are widely renowned tools utilizing rather simple measurement media and detection principles. Similarly, optically transparent and semi-transparent media can be used as cheap, consumable, readily available, small sample and technical expertise requiring materials that can be combined with basic detection principles, which are applying spectrophotometric measurements, as essential methods of many laboratories. Following this purpose, cellulose dialysis tubing (dialysis membrane) was started to be investigated for UV spectroscopy measurements of dry biological sample spots, with bovine serum albumin protein as the sample of interest. Dialysis membranes are among the common media in biological research and biosensor applications, but they have not been studied up to now, as the chief scaffold medium for sample drying and subsequent detection, by using UV-visible spectrophotometry. Hence this ongoing research focuses on exploring the feasibility of dialysis membrane as a novel platform for biological testing. The approach is also promising as a technique to be developed for point-of-care diagnosis since the necessary instruments of the proposed biosensor are appreciably miniaturized and can be listed among the mobile phone utilities in the future.
\end{abstract}

Keywords: Biosensor; dialysis membrane; bovine serum albumin; UV Spectrophotometry 


\section{Introduction}

Utilizing conventional media and measurement tools as distinct platforms for novel sensing and detection approaches are indispensable tools to biosensors research [1]. Cellulose-based nontransparent media, namely papers, have been utilized in biosensor studies with emphasis on microfluidics and colorimetric detection [2]. As a related research material, cellulose dialysis tubing (dialysis membrane) is among those tools, which are widely used, not only in medicine and biology, but in biosensor studies as well [3,4]. Their use in sensor applications is generally composed of improving the efficiencies and functionalities of the systems and devices [3,4]. They can even serve potentially as immobilization and adsorption media [5]. To the best of our knowledge, using them principally as a scaffold for sample preparation atop, for the forthcoming sample detection, by using established techniques such as UV-visible spectrometry is new to the field. Here, the presented work shows the implementation and proof of the concept with bovine serum albumin protein, as a biological sample. Moreover, $201 \mathrm{~nm}$ and $280 \mathrm{~nm}$ absorbance peaks were analyzed for the concentration dependent changes in the relevant spectral absorbencies, and the effect of varying sample spot sizes of the sample of interest was tested by changing the volume of the sample to be dried on the surface. Eventually, this work shares common features of the relevant media that are already in use, such as colorimetric detection of proteins [6] and paper-based microfluidics [2]. Therefore, similar principles are valid for the presented approach in this paper, which will be stimulating further studies in the future.

\section{Experimental Section}

Dialysis membrane (Sigma-Aldrich, MWCO 12400) was chosen as a readily available, cheap, disposable, and biocompatible medium to be studied. Spectrophotometric measurements were performed with Agilent 8453 UV-visible Spectroscopy System. Dialysis membrane is semitransparent in the region of interest, but influence of dialysis membrane absorbance on the proteinspecific absorbance was eliminated by performing reference measurements with the membrane, which was attached on the quartz spectrometer cuvette (Fig. 1(a)).

Different concentrations of bovine serum albumin protein (Sigma-Aldrich) sample solutions, ranging between $0.5 \mathrm{nM}$ to $500 \mu \mathrm{M}$ final concentrations, with 10 folds concentration changes among each sample, were prepared by using distilled water. Then, measurements of both distilled water and varying concentrations of bovine serum albumin sample solution was performed within the $190 \mathrm{~nm}-$ $1100 \mathrm{~nm}$ range, after preparing dried sample spots with these sample solutions, on the dialysis membrane, in the mid position of the UV-light passage hole of the instrument's cuvette holder. Samples were dried on the surface, at ambient atmospheric conditions, and became the spots to be measured with the spectrometer. Appearance of the transparent versus white-colored spots on the dialysis membrane is shown in Fig. 1(b), while the membranes are attached to the transparent, front sides of the cuvettes. Dialysis membrane was renewed before each measurement. 


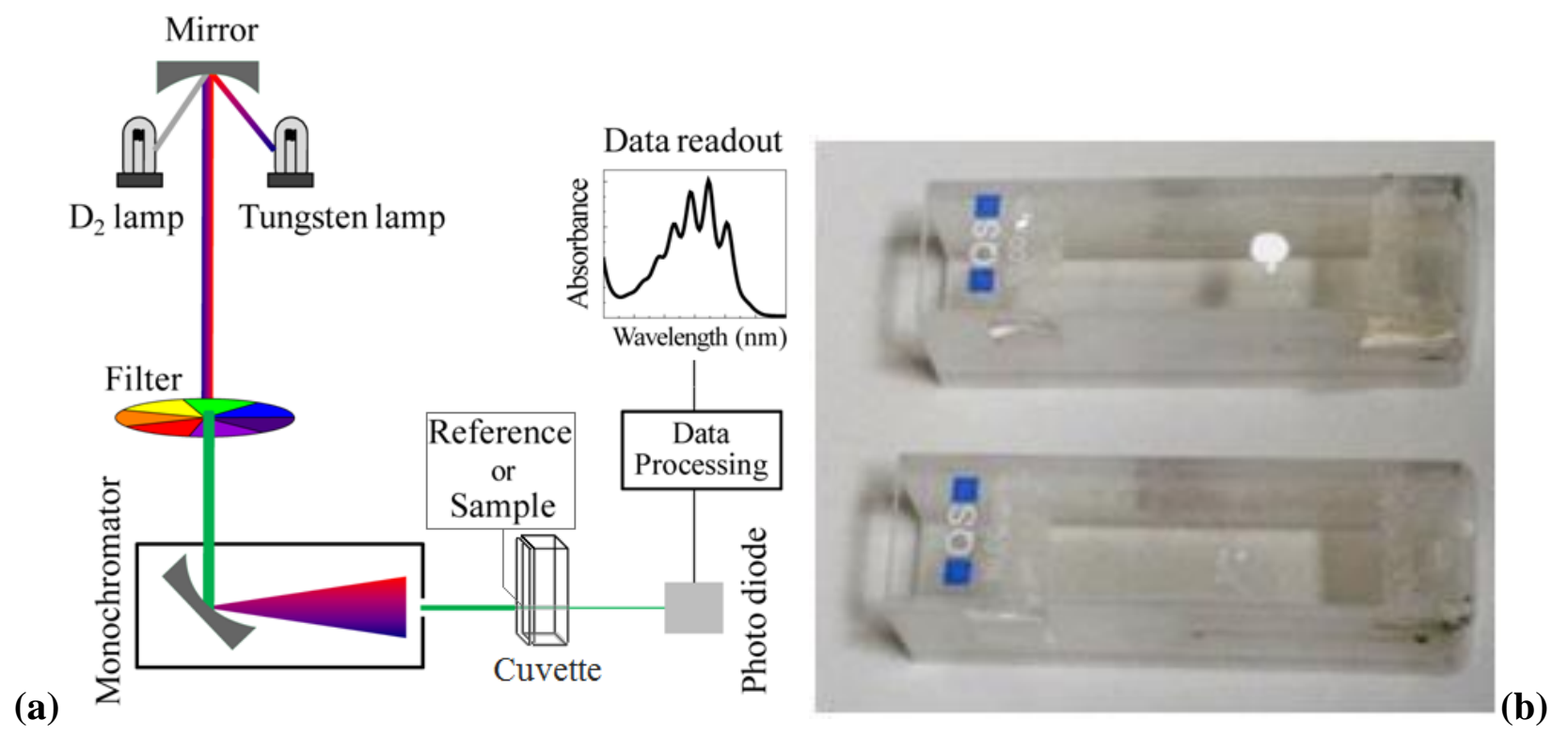

Fig. 1: Measurement scheme depiction (a) and photograph of dried-sample bearing dialysis membranes that are attached on the sample cuvettes of the UV spectrophotometer (b). In measurement scheme depiction (a), reference is the dialysis membrane without sample and sample is the dried sample spot on the same dialysis membrane. Image in (a) was adapted from the Wiki file entitled "Schematic of UV- visible spectrophotometer" [7]. In (b), the photograph shows the appearance of the transparent (at the bottom) versus white-colored (on top) spots on the dialysis membranes, which are attached to the transparent, front sides of the cuvettes.

Dialysis membrane is hydrophilic, which leads to the fact that the aqueous media have the tendency to spread on the membrane surface. Therefore, two different sample volumes were used for the measurements, which were $0.5 \mu \mathrm{l}$ and $2 \mu \mathrm{l}$, with the purpose of increasing the final dry spot size of the sample. The selected wavelengths for analysis were $201 \mathrm{~nm}$ and $280 \mathrm{~nm}$. These wavelengths were selected with respect to the measurements of the bovine serum albumin protein, both as a dried sample spot and in solution, with varying concentrations between $0.5 \mathrm{nM}$ to $500 \mu \mathrm{M}$. Relevant data is shown below, in "Results and Discussion" section, only for dried sample spot measurements that were prepared by using $0.5 \mu \mathrm{M}, 5 \mu \mathrm{M}, 50 \mu \mathrm{M}$, and $500 \mu \mathrm{M}$ protein sample solutions.

\section{Results and Discussion}

\subsection{Effect of Wavelength Selection on Concentration-Dependent Absorbance}

As stated above, $201 \mathrm{~nm}$ and $280 \mathrm{~nm}$ were the selected wavelengths, for analysis of the spectral data. According to the results, which are shown below, $280 \mathrm{~nm}$ is the most representative wavelength (Fig. 2 and Fig. 3). On the other hand, $201 \mathrm{~nm}$ is overlapping with a broad spectral feature, which makes it harder to analyze the spectra quantitatively, and also for the spectral shifts. Still, the selected absorbance values were increasing with the concentrations of bovine serum albumin sample solutions, which were dried on the dialysis membrane surfaces (Fig. 2). This supports the applicability of the method for protein detection and concentration determination. 


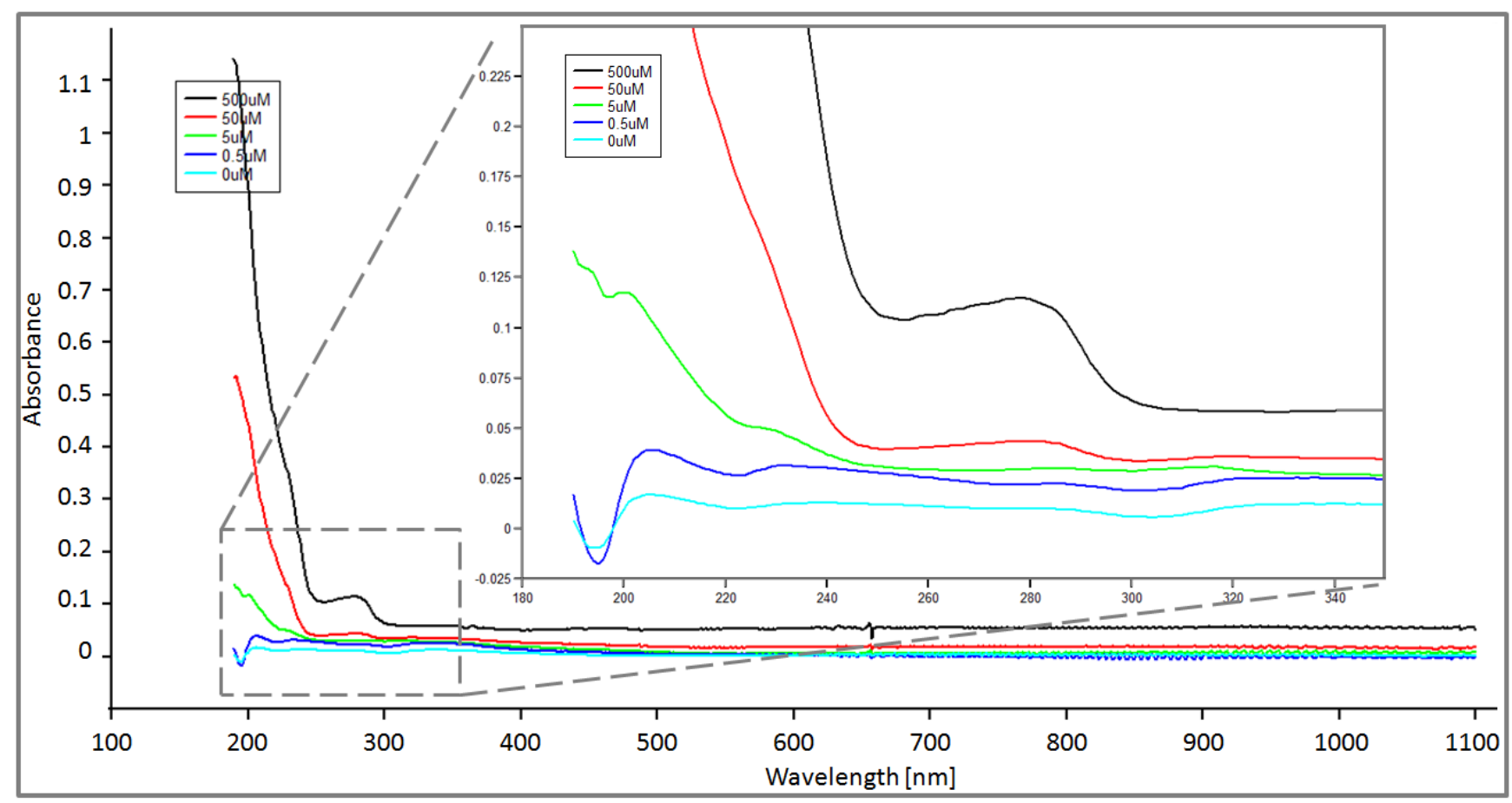

Fig. 2: Sample spectra. UV-visible spectra of sample spots that were prepared by drying $0.5 \mu 1$ of distilled water (shown as $0 \mu \mathrm{M}$ ), and $0.5 \mu \mathrm{M}, 5 \mu \mathrm{M}, 50 \mu \mathrm{M}$, and $500 \mu \mathrm{M}$ bovine serum album protein. Inset shows the zoom in view of 190-350 nm wavelength and -0.025-0.225 absorbance region. Graphics were generated by the freeware optical spectroscopy software, Spekwin32 (version 1.71.6.1), which was developed by Dr. Friedrich Menges.

\subsection{Effect of Sample Spot Size}

As mentioned above in the "Experimental Section", initial sample volume, which was $0.5 \mu 1$, was increased four times in a second testing scheme, to enhance the final dry spot size on the hydrophilic membrane. Accordingly, $201 \mathrm{~nm}$ (Fig. 3(a)) and $280 \mathrm{~nm}$ (Fig. 3(b)) absorbencies of spectra that were obtained by measuring dried spots of $0.5 \mu 1$ and $2 \mu 1$ volume samples are shown comparatively on the same graphs, in Fig. 3. Increasing the sample volume resulted in increased amount of sample on the surface and eventually, elevated absorbance signals at both $201 \mathrm{~nm}$ (Fig. 3(a)) and $280 \mathrm{~nm}$ (Fig. 3(b)). However, this did lead to the prolongation of drying duration of the sample on the surface, from less than 5 minutes to more than 10 minutes. Besides, sample preparation requires further optimization, to improve the initial positive results. For instance, membrane texture prevents even dissipation of the aqueous sample, as a circular spot. Negative influence of this situation needs to be eliminated or diminished, at least.

Fig. 3 also reveals that $280 \mathrm{~nm}$ absorbance enables better projection of the concentration dependent absorbance of dry protein sample spots, regardless of the volume of the sample that has been used. This is based on the fact that linearity of spectral absorbencies at $280 \mathrm{~nm}$ (Fig. 3(b)) for concentrations above $5 \mu \mathrm{M}$ is good for both of the utilized volumes, contrary to that at $201 \mathrm{~nm}$ (Fig. 3(a)). 


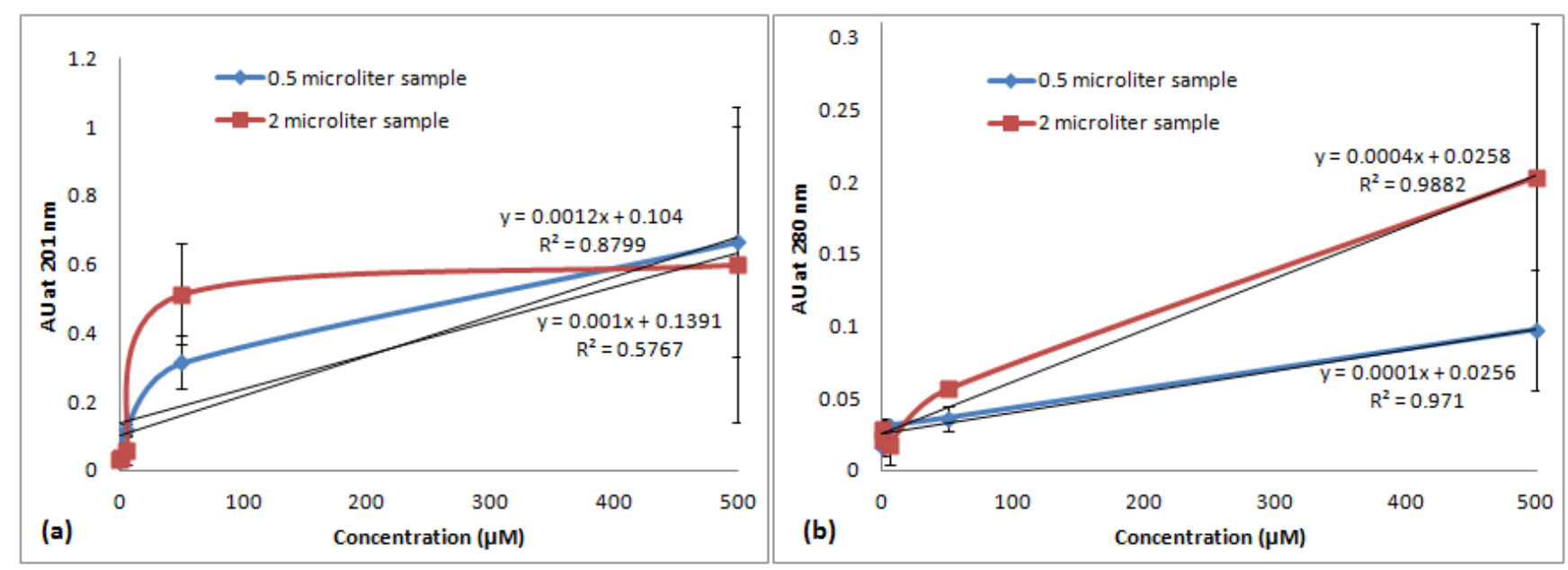

Fig. 3: Effect of sample volume and resulting dry-spot size on $201 \mathrm{~nm}$ and $280 \mathrm{~nm}$ absorbencies (AU), at different sample concentrations. The graphs demonstrate $201 \mathrm{~nm}$ (a) and $280 \mathrm{~nm}$ (b) absorbencies of the sample spots that were prepared by drying either $0.5 \mu \mathrm{l}$ or $2 \mu \mathrm{l}$ of distilled water (shown as $0 \mu \mathrm{M}$ ), and $0.5 \mu \mathrm{M}, 5 \mu \mathrm{M}, 50 \mu \mathrm{M}$, and $500 \mu \mathrm{M}$ bovine serum albumin protein. Absorbance values were obtained by using the UV-Visible ChemStation Software of Agilent Technologies and graphics were generated by the Microsoft Office Excel program.

Among the tested samples, sample concentrations below $5 \mu \mathrm{M}$ could not be distinguished well from each other and this necessitates additional tests and analysis. However, it is not a drawback for the proposed method, as the ultimate aim is to measure proteins' and specific biomolecules' signals of undiluted biological specimens, which rather have higher protein concentrations. Still, the standard deviations were found to be high, which indicates that improvements and attaining consistency in the sampling procedure and test set-up are required.

\section{Conclusions/Outlook}

Dialysis membrane is used for the first time, in order to detect dried protein sample spots, by UV spectrophotometry. As mentioned, dialysis membrane was renewed before each measurement. Nevertheless, correlation of the absorbencies at $201 \mathrm{~nm}$, and especially at $280 \mathrm{~nm}$, with the concentration of the protein sample that was dried on the surface, proves the applicability of the method. This was achieved by using both $0.5 \mu 1$ and $2 \mu 1$ of samples for the tests. Considering possible adverse effects of parameter variations among the tests, observing concentration dependency in the current measurement scheme is somehow indicative of robustness.

Measurement of real biological samples with the same technique is currently under investigation. Yet, sample preparation requires further optimization, along with necessary developments and establishing reproducibility of the test results. In addition to these work, which awaits for bringing the test results to high grade standards, the presented approach is fruitful in the sense that implementation of colorimetric assays for improving signal qualities and quantities, and adaption of microfluidic testing schemes with paper-based microfluidics, are potential novel research areas that can be nourished from this work. Last, as mentioned in the very beginning, optical measurement scheme of the proposed system paves the grounds for transforming it as a mobile phone utility as well. 


\section{Acknowledgments}

The author acknowledges Assoc. Prof. Dr. Cengiz Ozalp, for providing dialysis membrane sample; and Istanbul Kemerburgaz University, for providing the lab facilities and remaining consumables.

\section{Author Contributions}

Yekbun Adiguzel performed the presented work, including experimental design, data analysis, interpretation of the results, and manuscript preparation.

\section{Conflicts of Interest}

The author declares no conflict of interest.

\section{References and Notes}

1. Adiguzel, Y.; Kulah, H. Studies on visual detection and surface modification testing of glaff microfiber filter paper based biosensor. Biosens. Bioelectron. 2014, 54, $27-34$.

2. Liana, D.D.; Raguse, B.; Gooding, J.J.; Chow, E. Recent Advances in Paper-Based Sensors. Sensors 2012, 12, 11505-11526.

3. Tkac, J.; Vostiar, I.; Gorton, L.; Gemeiner, P.; Sturdik, E. Improved selectivity of microbial biosensor using membrane coating. Application to the analysis of ethanol during fermentation. Biosens. Bioelectron. 2003, 18, 1125-1134.

4. Petrou, P.S.; Moser, I.; Jobst, G. Microdevice with integrated dialysis probe and biosensor array for continuous multi-analyte monitoring. Biosens. Bioelectron. 2003, 18, 613-619.

5. Nguyen, Q.T.; Glinel, K.; Pontié, M.; Ping, Z. Immobilization of bio-macromolecules onto membranes via an adsorbed nanolayer - An insight into the mechanism. J. Memb. Sci. 2004, 232, 123-132.

6. Islam, M.R.; Serpe, M.J. Label-free detection of low protein concentration in solution using a novel colorimetric assay. Biosens. Bioelectron. 2013, 49, 133-138.

7. File:Schematic of UV- visible spectrophotometer.png. Available online: http://commons.wikimedia.org/wiki/File:Schematic_of_UV__visible_spectrophotometer.png (accessed on 14 May 2014).

(C) 2014 by the author; licensee MDPI, Basel, Switzerland. This article is an open access article distributed under the terms and conditions of the Creative Commons Attribution license (http://creativecommons.org/licenses/by/3.0/). 